\title{
Analysis of mutations of $M D R 3$ exons 9 and 23 in infants with parenteral nutrition-associated cholestasis
}

\author{
XIU-FANG YANG ${ }^{1}$, GUO-SHENG LIU ${ }^{2}$ and MIN-XU LI ${ }^{3}$ \\ ${ }^{1}$ Department of Neonatology, Zhongshan People's Hospital Affiliated to Sun Yat-sen University, Zhongshan, \\ Guangdong 528403; ${ }^{2}$ Department of Neonatology, The First Affiliated Hospital of Jinan University, Guangzhou, \\ Guangdong 510630; ${ }^{3}$ Dongguan Maternal and Child Health Center, Dongguan, Guangdong 523000, P.R. China
}

Received May 13, 2014; Accepted January 8, 2015

DOI: $10.3892 /$ etm.2015.2800

\begin{abstract}
The aim of this study was to investigate mutations of multidrug resistance 3 (MDR3) exons 9 and 23 in infants with parenteral nutrition-associated cholestasis (PNAC). A total of 41 infants with PNAC were enrolled in the study. Genomic DNA was extracted from the peripheral venous blood leukocytes of each patient and $M D R 3$ exons 9 and 23 were amplified by polymerase chain reaction. One patient was identified who carried a frameshift mutation in MDR3 exon 23 (C.2793) that was caused by the insertion of a single adenine residue, while mutations were not found in MDR3 exon 23 in the other 40 patients. The clinical features of the patient with the MDR3 exon 23 frameshift mutation included high serum $\gamma$-glutamyl transferase levels, the absence of biliary dilatation and deformity in magnetic resonance cholangiopancreatography, and abnormal electrical capacitance tomography imaging of the liver. No mutations in $M D R 3$ exon 9 were identified in any of the patients. All 41 PNAC patients recovered following oral ursodeoxycholic acid treatment. The C.2793 frameshift mutation in MDR3 exon 23 is potentially associated with the development of PNAC in infants.
\end{abstract}

\section{Introduction}

Since Dudrick et al first reported the application of parenteral nutrition (PN) in newborns in 1968 (1), the prognosis of preterm infants has markedly improved. However, in 1971, Peden et al reported the case of a premature infant who developed severe liver function damage owing to the application of total parenteral nutrition (TPN) (2). The autopsy of this patient revealed the presence of intrahepatic cholestasis, bile duct dilatation

Correspondence to: Dr Guo-Sheng Liu, Department of Neonatology, The First Affiliated Hospital of Jinan University, 613 Huangpu West Road, Tianhe, Guangzhou, Guangdong 510630, P.R. China

E-mail: guoshengliudoc@163.com

Key words: parenteral nutrition associated-cholestasis, multidrug resistance 3 gene, mutation, clinical features and cirrhosis. Since then, parenteral nutrition-associated cholestasis (PNAC) in preterm infants has garnered increasing attention.

The pathogenesis of PNAC has not been elucidated until recently. PNAC is considered to be caused by a variety of factors, including premature birth, low birth weight, long PN duration, a lack of fasting, gastrointestinal irritation, infection, intestinal bacterial overgrowth, bacterial translocation, TPN solution nutrient imbalances, lack of trace elements and toxic ingredients in PN $(3,4)$. These factors cause liver damage, degeneration, and fat deposition in the liver $(5,6)$. In recent years, a number of studies have shown that MDR3 mutations or the decreased expression or dysfunction of $M D R 3$ causes bile phospholipid deficiency, bile stone formation and the obstruction of small bile ducts, and may affect bile metabolism, thereby causing cholestasis $(7,8)$. The purpose of the present study was to investigate whether mutations of $M D R 3$ exons 9 and 23 were present in infants with PNAC.

\section{Materials and methods}

Subjects. A total of 41 infants with PNAC were enrolled in the study between June 2011 and December 2013. PNAC diagnostic criteriaincluded: $\mathrm{PN}$ for $>14$ days, jaundice, a direct bilirubin level of $>1.5 \mathrm{mg} / \mathrm{dl}$, discolored stools, elevated liver enzymes, and the exclusion of biliary atresia, choledochal cysts, bile duct dilatation, surgery-induced disease, viral infection (hepatitis A, B or C and cytomegalovirus) and metabolic diseases (9).

Informed consent for participation in the study was received from all the subjects' guardians, and the protocol was approved by the ethics committee of the Zhongshan People's Hospital Affiliated to Sun Yat-Sen Univeristy (Zhongshan, China).

Study design. Blood cultures were routinely checked for cytomegaloviruses, syphilis and Toxoplasma gondii, and the blood levels of glucose, C-reactive protein and indicators of liver function [alanine aminotranferase (ALT), aspartate aminotransferase (AST), $r-\gamma$-glutamyl transferase (r-GGT), total bilirubin, direct bilirubin and total bile acid] were measured weekly. Genomic DNA was extracted from the peripheral venous blood leukocytes collected from 
Table I. Primers for MDR3 gene exons 9 and 23, annealing temperature and amplified fragment length.

\begin{tabular}{|c|c|c|c|c|}
\hline Exon & Primer direction & Primer sequence & $\begin{array}{l}\text { Annealing } \\
\text { temperature }\end{array}$ & $\begin{array}{l}\text { Length } \\
\text { of product }\end{array}$ \\
\hline \multirow[t]{2}{*}{ Exon 9} & Forward & 5'-GGGTTCATTACCTTGACTGAC-3' & \multirow[t]{2}{*}{$58^{\circ} \mathrm{C}$} & \multirow[t]{2}{*}{433 bp } \\
\hline & Reverse & 5'-CTGGACAGTGGAAAGATTCAC-3' & & \\
\hline \multirow[t]{2}{*}{ Exon 23} & Forward & 5'-AGCCGTGCTCTTTCCACT-3' & \multirow[t]{2}{*}{$54^{\circ} \mathrm{C}$} & \multirow[t]{2}{*}{$329 \mathrm{bp}$} \\
\hline & Reverse & 5'-ATCCCTGACCTCATCTTTGG-3' & & \\
\hline
\end{tabular}

each patient. Samples were stored at $-80^{\circ} \mathrm{C}$. Genomic DNA was extracted using the phenol-chloroform method (10). Polymerase chain reaction (PCR) was used to amplify MDR3 exons 9 and 23. Mutations in MDR3 exons 9 and 23 were detected using a restriction enzyme digestion method and by sequencing analysis using an ABI 3100 sequencer (Applied Biosystems, Foster City, CA, USA). The DNA sequences of each patient were compared with the human genome sequence for MDR3. The patients were examined by electrical capacitance tomography (ECT) with the intravenous injection of $\mathrm{Tc}^{99 \mathrm{~m}}$-ethyl hepatic iminodiacetic acid (EHIDA; $5 \mathrm{mCi}$ ), and also by magnetic resonance cholangiopancreatography (MRCP) and hepatobiliary ultrasound.

Materials and genetic analysis. DNAzol BD reagent (Invitrogen Life Technologies, Carlsbad, CA, USA), isopropanol, 75\% ethanol, QL-901 Vortex Shaker (Kylin-Bell Lab Instruments Co., Ltd, Haimen, China) and a highspeed centrifuge (Eppendorf, Hamburg, Germany) were used for the extraction of genomic DNA. The extracted DNA was examined and primers were synthesized by PCR-restriction fragment length polymorphism (PCR-RFLP), using a PCR machine (C1000; Bio-Rad Laboratories,Inc., Hercules, CA, USA). The primers were designed using Primer 5 software (Premier Biosoft, Palo Alto, CA, USA). PCR was used to amplify exon 9 and 23 of the $M D R 3$ gene. Each PCR reaction mixture contained $45 \mu 1$ Platinum PCR SuperMix (Invitrogen Life Technologies), $1 \mu 1$ forward primer $(10 \mu \mathrm{M}), 1 \mu \mathrm{l}$ reverse primer $(10 \mu \mathrm{M}), 2 \mu \mathrm{l}$ template DNA and $\mathrm{ddH}_{2} \mathrm{O}$. The primers for $M D R 3$ exons 9 and 23 and their respective annealing temperatures are shown in Table I. PCR conditions included an initial denaturation step at $94^{\circ} \mathrm{C}$ for $3 \mathrm{~min}$, followed by 30 cycles of denaturation at $94^{\circ} \mathrm{C}$ for $15 \mathrm{sec}$, and annealing at $72^{\circ} \mathrm{C}$ for $30 \mathrm{sec}$. The PCR reaction was terminated after an extension step at $72^{\circ} \mathrm{C}$ for $5 \mathrm{~min}$. The PCR products were observed following $1 \%$ agarose electrophoresis (Bio-Rad Laboratories, Inc.) using an automatic Bio-Rad Gel Doc ${ }^{\mathrm{TM}}$ XR gel imaging system (Bio-Rad Laboratories, Inc.) and a DL2000 DNA marker (Takara Biotechnology Co., Ltd., Dalin, China). MDR3 DNA was amplified by PCR (Bio-Rad Laboratories, Inc.), and the mutations of MDR3 exons 9 and 23 were detected using the restriction enzyme (sex AI) (Bio-Rad Laboratories, Inc.) digestion method, using an ABI Prism 3100 sequencer (Applied Biosystems). For the DNA sequence analysis, the DNA sequence were compared with the human genome sequence of the MDR3 gene in Genbank (http://www.ncbi. nlm.nih.gov/Genbank; accessed 01/06/2011). Gene muta-

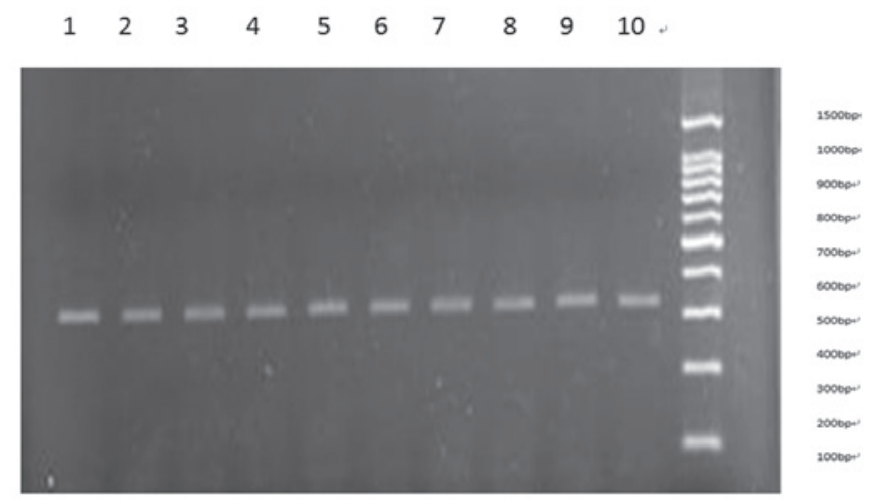

Figure 1. Amplified fragments of MDR3 gene exon 9. Lanes 1-9, amplified fragments of $M D R 3$ gene exon 9 of parenteral nutrition-associated cholestasis infants; M, DNA marker DL 2000.

1234567891011121314151617181920212223.

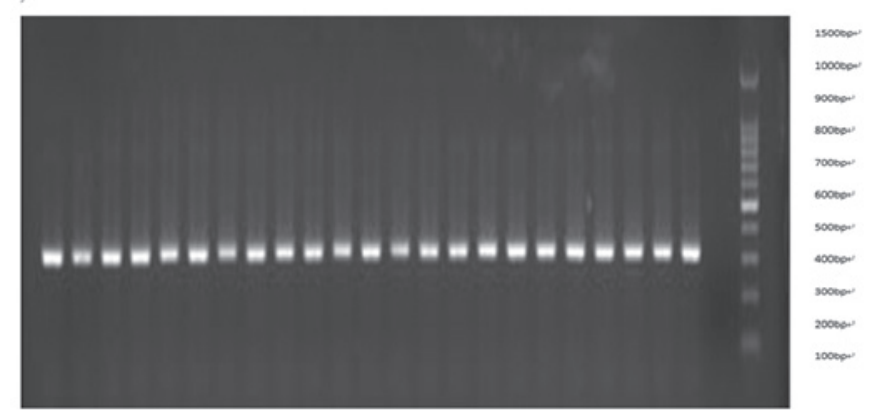

Figure 2. Amplified fragments of $M D R 3$ gene exon 23. Lanes 1-9, amplified fragments of $M D R 3$ gene exon 23 of parenteral nutrition-associated cholestasis infants; M, DNA marker DL 2000.

tions were analyzed using BioEdit protein contrast software obtained from www.piaodown.com (accessed 30/08/2011).

\section{Results}

Electrophoresis of PCR-amplified MDR3 fragments. The PCR-amplified fragments of MDR3 exons 9 and 23 were clearly visible when electrophoresis was performed. The product lengths were as expected (Figs. 1 and 2).

DNA sequencing. DNA sequencing analysis revealed that there was only one patient with a frameshift mutation in $M D R 3$ exon 23. This mutation involved the insertion of an adenine residue at position 2,793 (C.2793 mutation; Fig. 3). 
Table II. Comparison between the patient with a C.2793 frameshift mutation and the group without mutation.

\begin{tabular}{lcccccc}
\hline Group & No. of cases & ALT $(\mathrm{U} / \mathrm{l})$ & T-BIL $(\mu \mathrm{mol} / \mathrm{l})$ & D-BIL $(\mu \mathrm{mol} / \mathrm{l})$ & TBA $(\mu \mathrm{mol} / \mathrm{l})$ & $\mathrm{r}-\mathrm{GGT}(\mathrm{U} / \mathrm{l})$ \\
\hline With mutation & 1 & 140 & 150.3 & 80.2 & 90.6 & 116 \\
Without mutation & 40 & $119 \pm 19.8$ & $126.2 \pm 22.3$ & $69.7 \pm 10.3$ & $73.9 \pm 11.2$ & $59 \pm 5$ \\
\hline
\end{tabular}

The values of indicators of liver function for the without mutation group are presented as mean \pm standard deviation. ALT, alanine aminotransferase; T-BIL, total bilirubin; D-BIL, direct bilirubin; TBA, total bile acid; r-GTT, r- $\gamma$-glutamyl transferase.

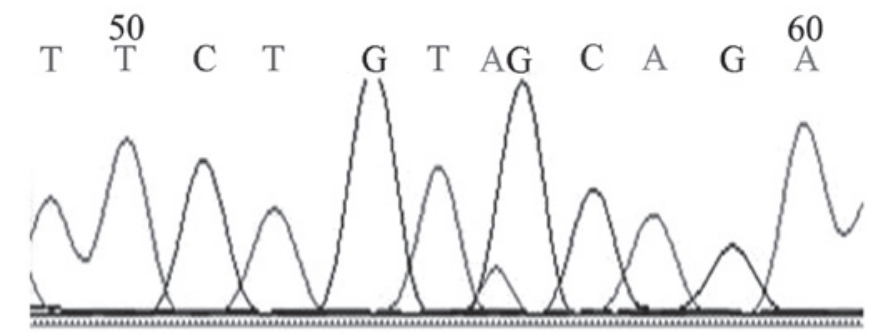

Figure 3. DNA sequencing of the MDR3 gene exon 23 frameshift mutation (one case).

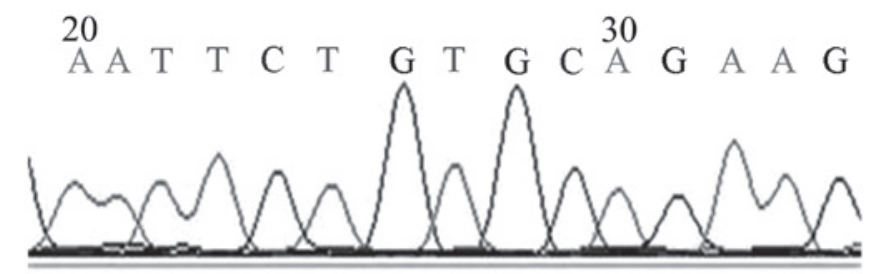

Figure 4. DNA sequencing of the MDR3 gene exon 23 with no mutation (40 cases).

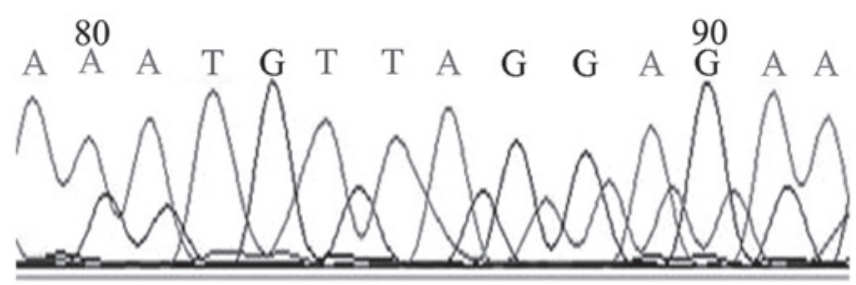

Figure 5. DNA sequencing of the MDR3 gene exon 9 with no mutation (41 cases).

$M D R 3$ exon 23 mutations were not identified in the other 40 infants with PNAC (Fig. 4). No MDR3 exon 9 mutations were identified in any of the 41 infants with PNAC that were included in this study (Fig. 5).

Clinical features. The levels of ALT, total bilirubin, direct bilirubin, total bile acid and r-GGT in the patient with the C.2793 frameshift mutation in MDR3 exon 23 were higher than those in the other patients who did not carry the mutation (Table II).

When the patient with the mutation was examined by ECT, the position of the liver was normal and the distribution of radioactivity in the liver was sparse. The results showed that there was no development in the gallbladder, biliary tract and intestines, whereas there was obvious development in the kidney and bladder. Hepatobiliary dynamic ECT imaging indicated that the excretion function of the liver was abnormal (Fig. 6). MRCP revealed that the morphology, size and lobes of the liver were normal. No abnormal signals were observed in the liver. Dilatation of the intrahepatic and extrahepatic bile ducts and the pancreatic duct was not observed. The gall bladder was normal and no signs of biliary calculi were present. The size and morphology of the pancreas and spleen were normal (Fig. 7). Hepatobiliary ultrasound showed that the size of the liver was normal and no biliary dilatation and deformity were observed in the patient with the MDR3 exon 23 frameshift mutation (Fig. 8). All 41 PNAC patients recovered following oral treatment with ursodeoxycholic acid $(10 \mathrm{mg} / \mathrm{kg} /$ day). The course of treatment was $\sim 125 \pm 12$ days.

\section{Discussion}

With the development of neonatology, the survival rate of premature infants has increased significantly. TPN has played an important role in this improvement (11); however, with the wide application of PN, the prevalence of PNAC in infants has increased. Severely affected patients may develop malnutrition, biliary cirrhosis and liver failure, which may even result in mortality (12-14). With the development of genomics, mounting evidence indicates that genetic factors such as MDR3 mutations are important in the pathogenesis of intrahepatic cholestasis, including low phospholipid-associated cholelithiasis (14-16), progressive familial intrahepatic cholestasis type 3 (17-19), intrahepatic cholestasis of pregnancy (20-22), fibrosing cholestatic liver disease (23) and sclerosing cholangitis (24).

The formation and excretion of bile involves bile transport to basolateral hepatocytes and across biliary duct membranes. The most important aspect of bile dynamics is bile acid secretion from the blood into the bile duct. The active transport of bile acid within the liver and of soluble substances across canalicular hepatocyte membranes are the rate-limiting steps in bile formation. Active transport requires several ATP export pump proteins [ATP-binding-cassette (ABC)-transport proteins]. Bile salt transport is mediated by the bile salt export pump. The multidrug resistance phospholipid glycoprotein $M D R 3$ is a specialized ABC-transporter that serves as the primary phospholipid transporter through the tubular membranes (25-27).

ATP-binding cassette subfamily B member 4 (ABCB4) transporters have phosphatidylcholine floppase activity. They translocate phosphatidylcholine from the inner to the 


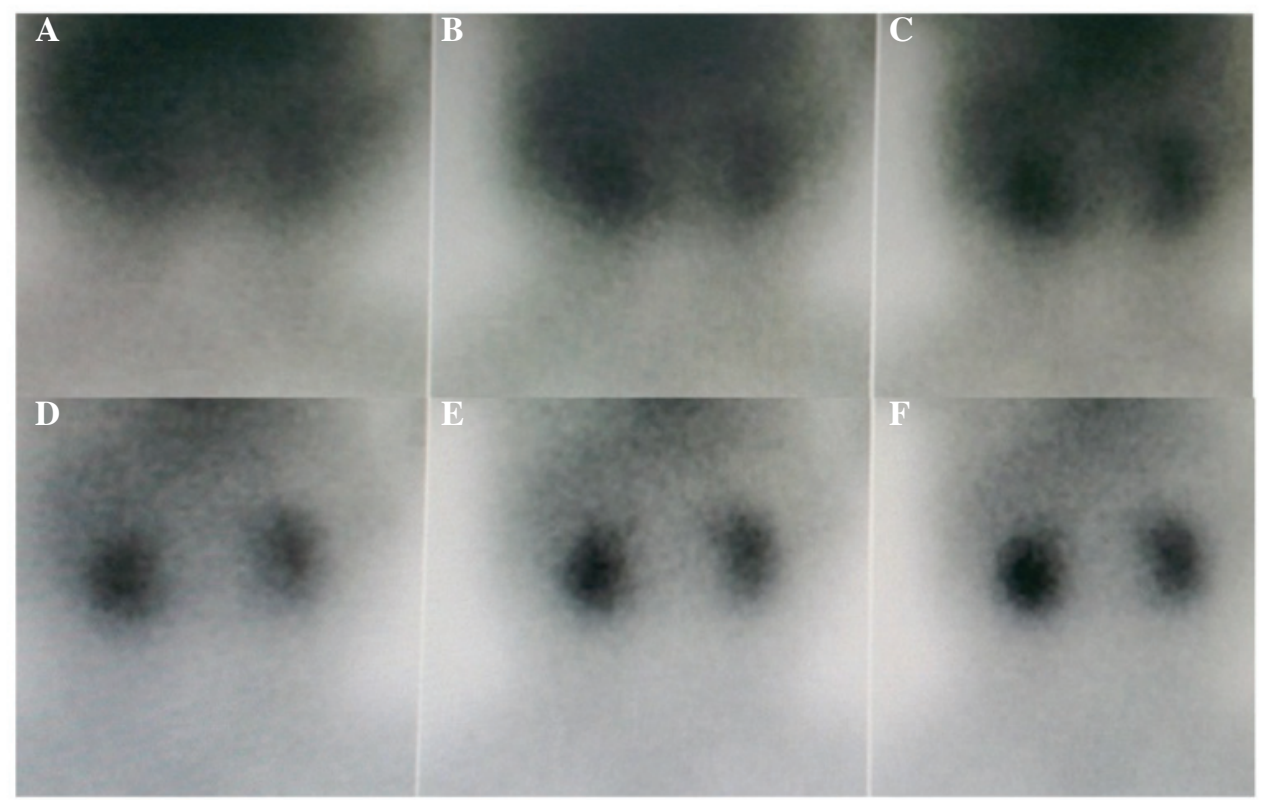

Figure 6. Electrical capacitance tomography imaging in the case with a C.2793 frameshift mutation in exon 23 of $M D R 3$ at (A) $30 \mathrm{~min}$, (B) $1 \mathrm{~h}$, (C) $2 \mathrm{~h}$, (D) $4 \mathrm{~h}$, (E) $6 \mathrm{~h}$ and (F) $24 \mathrm{~h}$ after intravenous Tc99m-EHIDA $5 \mathrm{mCi}$.

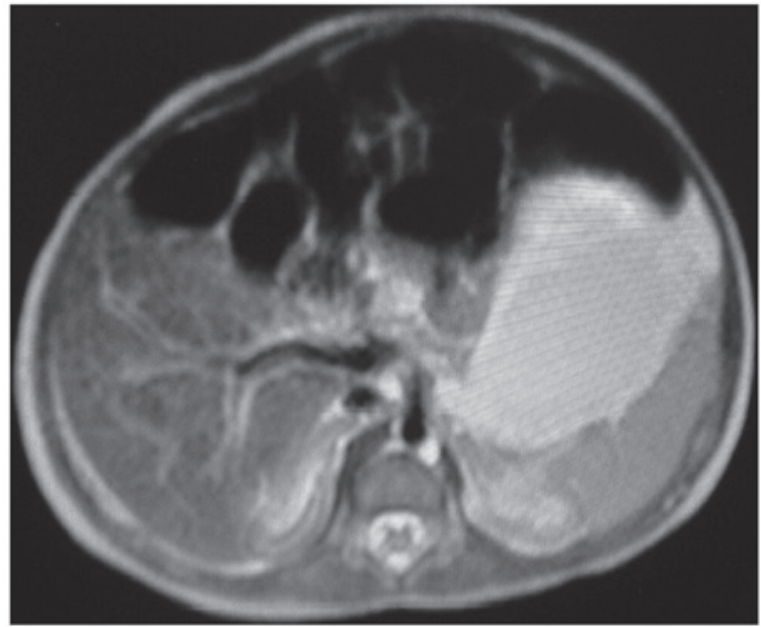

Figure 7. Magnetic resonance cholangiopancreatography (MRCP) imaging in the case with a C.2793 frameshift mutation in exon 23 of MDR3. MRCP revealed normal morphology, size and lobes of the liver, as well as no abnormal signals in the liver and no dilatation of the bile ducts.

outer leaflet of the canalicular membrane of hepatocytes, which renders phosphatidylcholine available for extraction into the canalicular lumen by bile salts. The role of $A B C B 4$ (MIM 171060) in this process is crucial, as demonstrated by the observation that its deficiency causes cholestatic liver diseases (28). The $A B C B 4$ gene, also known as $M D R 3$, encodes a member of the MDR/TAP subfamily that is associated with multidrug resistance and antigen presentation. In humans, $A B C B 4$ is located on chromosome 7q21.1, contains 27 coding exons and spans $\sim 74 \mathrm{~kb}$ (29). The pathophysiology associated with ABCB4 alterations relates to the lack of phospholipid protection from the detergent effect of bile salts, which results in damage to the biliary epithelium, bile ductular proliferation, and potential progressive portal fibrosis. Since

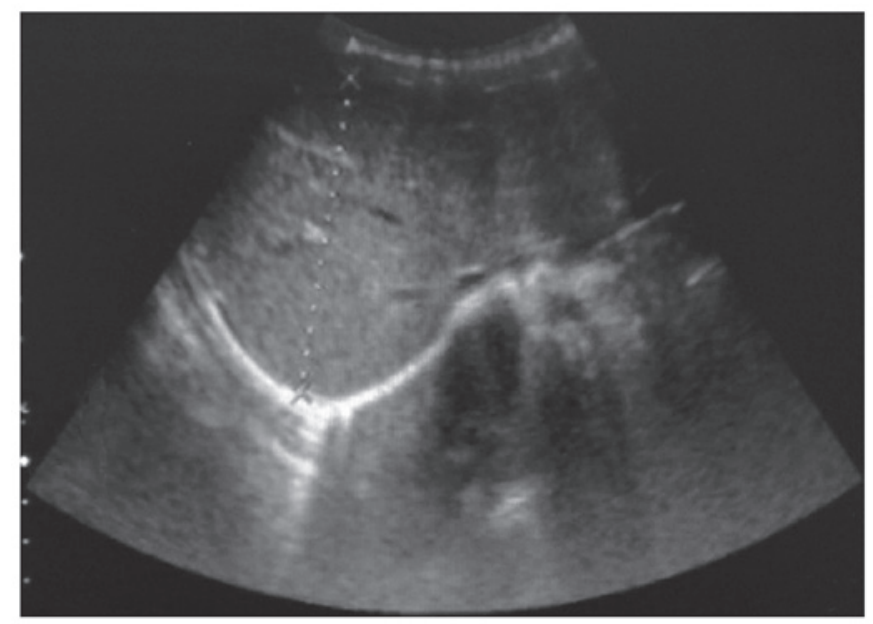

Figure 8. Hepatobiliary ultrasound in the case with a C. 2793 frameshift mutation in exon 23 of MDR3. Ultrasound showed normal size of the liver, no biliary dilatation or deformity.

the solubilization of biliary cholesterol depends on not only the concentration of the sterol, but also on the concentration of bile salt and phospholipid, a reduction in the rate of phospholipid excretion can also be a cause of gallstone formation. The reduced expression of $M D R 3$ reduces lecithin secretion and elevates vesicle cholesterol. It can also lead to bile duct damage, gallstone deposition, inflammation and biliary liver lesions (30-32).

The wide clinical spectrum of $A B C B 4$-deficiency syndromes in humans encompasses cholestatic disorders, presenting from the neonatal period of life to late adulthood (8). In the present study, DNA sequencing analysis of MDR3 exons 9 and 23 in 41 infants with PNAC identified only one patient who possessed a frameshift mutation in MDR3 exon 23. This mutation was characterized by the insertion of a single adenine at position 
2,793. MDR3 exon 23 mutations were not identified in the other 40 infants with PNAC. All 41 patients recovered following oral ursodeoxycholic acid treatment. The clinical features of the patient with the MDR3 exon 23 frameshift included high serum r-GT levels, the absence of biliary dilatation and deformity in MRCP, and abnormal ECT imaging of the liver. In the 41 PNAC patients, no MDR3 exon 9 mutations were found. The frameshift mutation caused by the insertion of an adenine residue at position 2,793 may be associated with the pathogenesis of PNAC in infants. However, the majority of infants with PNAC in this study did not carry a mutation in MDR3 exon 23 . Therefore, future studies are required to confirm the correlation between this frameshift mutation in MDR3 exon 23 and the incidence of PNAC in infants.

\section{References}

1. Dudrick SJ, Wilmore DW, Vars HM and Rhoads JE: Long-term total parenteral nutrition with growth development and positive nitrogen balance. Surgery 64: 134-142, 1968.

2. Peden VH, Witzleben CL and Skelton MA: Total parenteral nutrition. J Pediatr 78: 180-181, 1971.

3. Suchy FJ, Sokol RJ and Balistreri WF (eds): Approach to the infant with cholestasis. In: Liver Disease in Children. 2nd edition. Lippincott Williams and Wilkins, Philadelphia, pp187-194,2001.

4. Benchimol EI, Walsh CM and Ling SC: Early diagnosis of neonatal cholestatic jaundice: test at 2 weeks. Can Fam Physician 55: 1184-1192, 2009.

5. Baserga MC and Sola A: Intrauterine growth restriction impacts tolerance to total parenteral nutrition in extremely low birth weight infants. J Perinatol 24: 476-481, 2004

6. Kaufman SS, Gondolesi GE and Fishbein TM: Parenteral nutrition associated liver disease. Semin Neonatol 8: 375-381, 2003.

7. Hu HP, Zhou HB and Zhou DX: Molecular mechanism of bile formation and cholestatic diseases. Zhonghua Gan Zang Bing Za Zhi 16: 571-573, 2008 (In Chinese).

8. Oude Elferink RP and Paulusma CC: Function and pathophysiological importance of ABCB4 (MDR3 P-glycoprotein). Pflugers Arch 453: 601-610, 2007

9. Cholestatic Liver Disease Diagnosis and Treatment Expert Committee. Experts consensus on diagnosis and therapy of cholestasis. Chinese Journal of Experimental and Clinical Infectious Diseases (Electronic edition) 3: 474-487, 2009 (In Chinese).

10. Barnett R and Larson G: A phenol-chloroform protocol for extracting DNA from ancient samples. Methods Mol Biol 840: 13-19, 2012.

11. Pinto CardosoG,Abily-DonvalL,Chadie A, etal:Epidemiological study of very preterm infants at Rouen University Hospital: changes in mortality, morbidity, and care over 11 years. Arch Pediatr 20: 156-163, 2013 (In French).

12. Klein CJ, Revenis M, Kusenda C and Scavo L: Parenteral nutrition associated conjugated hyperbilirubinemia in hospitalized infants. J Am Diet Assoc 110: 1684-1695, 2010.

13. Robinson DT and Ehrenkranz RA: Parenteral nutrition-associated cholestasis in small for gestational age infants. J Pediatr 152: 59-62, 2008

14. Rafeey M, Golzar A and Javadzadeh A: Cholestatic syndromes of infancy. Pak J Biol Sci 11: 1764-1767, 2008.

15. Pasmant E, Goussard P, Baranes L, et al: First description of $\mathrm{ABCB} 4$ gene deletions in familial low phospholipid-associated cholelithiasis and oral contraceptives-induced cholestasis. Eur J Hum Genet 20: 277-282, 2012.
16. Poupon R, Barbu V, Chamouard P, Wendum D, Rosmorduc O and Housset C: Combined features of low phospholipid-associated cholelithiasis and progressive familial intrahepatic cholestasis 3 . Liver Int 30: 327-331, 2010.

17. Colombo C, Vajro P, Degiorgio D, et al: Clinical features and genotype-phenotype correlations in children with progressive familial intrahepatic cholestasis type 3 related to ABCB4 mutations. J Pediatr Gastroenterol Nutr 52: 73-83, 2011.

18. Degiorgio D, Colombo C, Seia M, et al: Molecular characterization and structural implications of 25 new ABCB4 mutations in progressive familial intrahepatic cholestasis type 3 (PFIC3). Eur J Hum Genet 15: 1230-1238, 2007.

19. van der Woerd WL, van Mil SW, Stapelbroek JM, Klomp LW, van de Graaf SF and Houwen RH: Familial cholestasis: progressive familial intrahepatic cholestasis, benign recurrent intrahepatic cholestasis and intrahepatic cholestasis of pregnancy. Best Pract Res Clin Gastroenterol 24: 541-553, 2010.

20. Wasmuth HE, Glantz A, Keppeler H, et al: Intrahepatic cholestasis of pregnancy: the severe form is associated with common variants of the hepatobiliary phospholipid transporter ABCB4 gene. Gut 56: 265-270, 2007.

21. Schneider G, Paus TC, Kullak-Ublick GA, et al: Linkage between a new splicing site mutation in the $M D R 3$ alias ABCB4 gene and intrahepatic cholestasis of pregnancy. Heptology 45: 150-158, 2007.

22. Harikar W, Kansal S, Oude Elferink RP and Angus P: Intrahepatic cholestasis of pregnancy: when should you look further? World J Gastroenterol 15: 1126-1129, 2009

23. Ziol M, Barbu V, Rosmorduc O, et al: BCB4 heterozygous gene mutations associated with fibrosing cholestatic liver disease in adults. Gastroenterology 135: 31-141, 2008.

24. Denk GU, Bikker H, Lekanne Dit Deprez RH, et al: ABCB4 deficiency: a family saga of early onset cholelithiasis, sclerosing cholangitis and cirrhosis and a novel mutation in the ABCB4 gene. Hepatol Res 40: 937-941, 2010.

25. Kullak-Ublick GA, Stieger B and Meier PJ: Enterohepatic bile salt transporters in normal physiology and liver disease. Gastroenterorlogy 126: 322-342, 2004.

26. Groen A, Romero MR, Kunne C, et al: Complementary functions of the flippase ATP8B1 and the floppase ABCB4 in maintaining canalicular membrane integrity. Gastroenterology 141: 1927-1937, 2011.

27. Karpen SJ: Transcriptional regulation of hepatobiliary transporters. In: Molecular Pathogenesis of Cholestasis. Trauner $\mathrm{M}$ and Jansen PLM (eds). Landes Bioscience, Georgetown, pp124-140, 2003.

28. OudeElferink RP,Paulusma CCand Groen AK: Hepatocanalicular transport defects: pathophysiologic mechanisms of rare diseases. Gastroenterology 130: 908-925, 2006.

29. Lincke CR, Smit JJ, van der Velde-Koerts T and Borst P. Structure of the human MDR3 gene and physical mapping of the human MDR locus. J Biol Chem 266: 5303-5310, 1991.

30. Gotthardt D, Runz H, Keitel V, et al: A mutation in the canalicular phospholipid transporter gene, $\mathrm{ABCB} 4$, is associated with cholestasis, ductopenia and cirrhosis in adults. Hepatology 48: 1157-1166, 2008

31. Wendum D, Barbu V, Rosomorduc O, Arrivé L, Fléjou JF and Poupon R: Aspects of liver pathology in adult patients with MDR3/ABCB4 gene mutations. Virchows Arch 460: 291-298, 2012.

32. Paulusma CC, Groen A, Kunne C, et al: Atp8b1 deficiency in mice reduces resistance of the canalicular membrane to hydrophobic bile salts and inpairs bile salt transport. Hepatology 44: 195-204, 2006. 